\title{
Variación espacio-temporal de la meiofauna submareal en una playa arenosa nororiental de Venezuela
}

\author{
Ildefonso Liñero Arana ${ }^{1}$, Sol Ojeda ${ }^{2} \&$ María Elena Amaro $^{1}$ \\ 1. Departamento de Biología Marina, Instituto Oceanográfico de Venezuela, Universidad de Oriente, Cumaná, \\ Venezuela; ilineroarana@yahoo.es, meamarao_2000@yahoo.com \\ 2. Departamento de Biología, Escuela de Ciencias, Universidad de Oriente, Cumaná, Venezuela; solojeda@yahoo.com
}

Recibido 07-XI-2011. Corregido 08-X-2012. Aceptado 06-XI-2012.

\begin{abstract}
Spatio-temporal variation of subtidal meiofauna in a sandy beach from Northeastern Venezuela. Meiofauna organisms that play an important role in the trophic ecology of soft bottom benthos, have short life cycles and they respond quickly to disturbance and pollution. The present study shows the spatio-temporal variation of subtidal meiofauna (metazoans passing a $500 \mu \mathrm{m}$ sieve but retained on meshes of $40-63 \mu \mathrm{m}$ ) in four shallow subtidal stations. Samples were taken in the sandy beach of San Luis, in the Northeastern coast of Venezuela, from October 2005 until September 2006. For this, three replicate sediment core samples $\left(4.91 \mathrm{~cm}^{2}\right)$, were collected monthly to a depth of $10 \mathrm{~cm}$ into the sediment, and preserved in $6 \%$ formalin stained with rose Bengal. Specimens of 14 meiofaunal groups (Foraminifera excluded) were collected, being the nematodes, ostracods and harpacticoid copepods the most abundant. Monthly density was comprised between 64 and 503 ind./10 $\mathrm{cm}^{2}$, and mean density of stations between 173 and $449 \mathrm{ind} . / 10 \mathrm{~cm}^{2}$. There is a trend of low densities from October to February (end of the rainy season until the middle of the dry season). The San Luis beach control of the meiofaunal community is shared by climatic conditions and by the biology of the species found. The meiofauna mean density in San Luis beach (263ind. $/ 10 \mathrm{~cm}^{2}$ ) was low when compared to other studies in tropical areas. Rev. Biol. Trop. 61 (1): 59-73. Epub 2013 March 01.
\end{abstract}

Key words: meiobenthos, nematodes, copepods, ostracods, Caribbean, Venezuela.

En las últimas décadas los estudios sobre la meiofauna se han intensificado debido a su valor en los monitoreos de contaminación orgánica (Raffaelli \& Mason 1981, Raffaelli 1987, Essink \& Keidel 1998) o química (Mahmoudi et al. 2005). Hay un consenso general en que la meiofauna es más apropiada que la macrofauna para los estudios de contaminación, debido a su abundancia, brevedad de sus ciclos de vida, reproducción continua a lo largo del año, ausencia de larvas planctónicas, estrecha relación con los sedimentos y facilidad de muestreo (Smol et al. 1991, Kennedy \& Jacoby 1997, Sandulli \& Grimaldi 2000). Son numerosos los factores que intervienen en la estructuración de las comunidades de la meiofauna: factores ambientales (corrientes, mareas, oleaje, temperatura, salinidad, entre otros) y las características granulométricas y químicas de los sedimentos (Giere 1993, Brazeiro 2001, Covazzi et al. 2001, Gheskiere et al. 2005, Díaz-Asencio et al. 2009), los procesos biológicos, como depredación, reproducción, ciclos de vida (Watzin 1985, Buffan-Dubau et al. 1996), estrategias alimenticias (Fisher \& Sheaves 2003), contaminación orgánica (Sandulli \& Grimaldi 2000, Gray et al. 2002, Fraschetti et al. 2006), eutrofización (Guiral et al. 1995, Andersen et al. 2006), cultivos acuáticos (La Rosa et al. 2001, Sutherland, et al. 2007) y bioperturbación (Warwick et al. 1990, Ólafsson \& Elmgren 1991, Rosa \& Bemvenuti 2005).

Las playas arenosas son ambientes físicamente estresados (Rodil \& Lastra 2004), en 
las cuales la abundancia y distribución de los organismos están sometidos principalmente a factores climáticos y a las características hidrodinámicas y de los sedimentos, y donde las interacciones biológicas no juegan un rol tan importante en la estructuración de las comunidades (McLachlan 1983). A estos factores se añade la perturbación ambiental ocasionada por las actividades turísticas, que pueden alterar notablemente a las poblaciones de la meiofauna (Gheskiere et al. 2005).

No obstante el creciente interés en la ecología de la meiofauna, son escasos los estudios realizados en el trópico americano. En la costa pacífica de Costa Rica existen los trabajos de De la Cruz \& Vargas (1986, 1987), Guzmán-Espinal et al. (1987) y Vargas (1988), principalmente referidos a las variaciones espaciales y temporales de la meiofauna costera de fondos blandos; en el Caribe se pueden citar los realizados en Cuba por Herrera \& Sánchez (1982), Armenteros et al. (2003), Pérez \& López-Cánovas (2006) en arrecifes coralinos de Cuba, Díaz-Asencio et al. (2009) estudiaron la meiofauna de fondos fangosos de la Bahía de Cienfuegos, Cuba, los de Renaud-Mornant \& Gourbault (1984), Boucher \& Gourbault (1990) en fondos costeros arenosos de la Isla de Guadalupe. En Jamaica el microfitobentos y el meiobentos fueron analizados por Edwards (2009). Gomes et al. (2002) y Netto \& Gallucci (2003) estudiaron la meiofauna asociada a manglares en Brasil. En Venezuela sólo se conocen los trabajos de Tietjen (1984) referido a especies de nemátodos de aguas profundas de la cuenca de Venezuela, y el de VásquezSuárez et al. (2010), quienes analizaron la variación temporal de la meiofauna en la Laguna de Raya, Isla de Margarita.

Tomando en consideración la escasez de estudios sobre la meiofauna en la costa sur del Mar Caribe, este estudio tiene como objetivo analizar la variación espacial y temporal de la estructura de la meiofauna dura en la playa de San Luis y establecer comparaciones con otros estudios similares realizados en zonas tropicales y subtropicales americanas.

\section{MATERIALES Y MÉTODOS}

Área de estudio: La ciudad de Cumaná está situada en la costa nororiental de Venezuela, en la costa sur de la entrada del Golfo de Cariaco. El clima es árido, con dos períodos climáticos, uno lluvioso, desde mayo hasta diciembre, que coincide con las mayores temperaturas; y un periodo seco en los meses restantes, con temperaturas inferiores y durante el cual los alisios soplan con intensidad (Quintero et al. 2004, Terejova et al. 2008).

La playa de San Luis, situada en la costa occidental de Cumaná, es un balneario de aproximadamente $5700 \mathrm{~m}$ situado entre las coordenadas $\left(10^{\circ} 26^{\prime} 40.33^{\prime \prime} \mathrm{N}-64^{\circ} 11^{\prime} 44.53^{\prime \prime}\right.$ W y $10^{\circ} 24^{\prime} 49.13^{\prime \prime} \mathrm{N}-64^{\circ} 14^{\prime} 07.72$ ' W, frecuentado durante todo el año, con mayor intensidad durante las vacaciones escolares entre julio y septiembre, durante los días de carnaval y en Semana Santa, particularmente en los sectores central y occidental, donde existen instalaciones hoteleras y clubes. Es una playa disipativa de pequeña amplitud de marea (promedio $30 \mathrm{~cm})$, ligera pendiente $\left(\leq 10^{\circ}\right)$ y baja energía, principalmente de mayo a noviembre. Presenta contaminación bacteriana de origen fecal y alta demanda de oxígeno, con incremento durante la época de lluvias (mayo a noviembre) principalmente en el sector oriental (Iabichella 1993), debido a la descarga de aguas domésticas y de un canal de recogida de aguas de lluvia al que incontroladamente vierten aguas servidas numerosas casas situadas en sus márgenes. En la zona central, existe un canal de comunicación entre la playa y la Laguna de Los Patos, y en la zona oeste desemboca el río Tacal, con un importante volumen de agua durante el periodo de lluvias.

Muestreo y extracción de la meiofauna: Mensualmente, desde octubre 2005 hasta septiembre 2006, en cuatro estaciones paralelas a la costa, a una profundidad de $1 \mathrm{~m}$, se tomaron tres réplicas de sedimento de $10 \mathrm{~cm}$ de profundidad con un nucleador de $2.5 \mathrm{~cm}$ de diámetro interno (volumen de cada muestra: $49.09 \mathrm{~cm}^{3}$ ). Las estaciones fueron seleccionadas evitando 
las zonas de mayor concurrencia de bañistas y, por lo tanto, de mayor perturbación antrópica, y estuvieron situadas en las siguientes coordenadas geográficas: Estación 1 (10²6'50" $\left.\mathrm{N}-64^{\circ} 11^{\prime} 38^{\prime \prime} \mathrm{W}\right)$, estación $2\left(10^{\circ} 25^{\prime} 27^{\prime \prime}\right.$ $\left.\mathrm{N}-64^{\circ} 12^{\prime} 47.83^{\prime \prime} \mathrm{W}\right)$, estación $3\left(10^{\circ} 25^{\prime} 07^{\prime}\right.$ ' $\left.\mathrm{N}-64^{\circ} 13^{\prime} 12^{\prime \prime} \mathrm{W}\right)$ y estación $4\left(10^{\circ} 24^{\prime} 58^{\prime \prime} \mathrm{N}\right.$ - 64'13'32” W), con una distancia de $3.19 \mathrm{~km}$ entre la E1 y E2, de $1 \mathrm{~km}$ entre la E2 y E3, y de $0.6 \mathrm{~km}$ entre la E3 y E4. Las muestras se fijaron in situ con formalina al 6\% en agua de mar teñida con rosa de Bengala. El sedimento fue tamizado con agua corriente a través de un tamiz de $500 \mu \mathrm{m}$ de abertura de malla, y el material filtrado fue lavado ocho veces a través de un tamiz de $63 \mu \mathrm{m}$ de malla (modificado de Pfannkuche \& Thiel 1988)

Los especímenes retenidos, excepto foraminíferos, fueron contados bajo observación con un microscopio estereoscópico Leica MZ95, a un aumento de 400X. Mediciones de la salinidad con un refractómeto manual de una unidad de apreciación y de la temperatura del agua, con un termómetro de $0.01{ }^{\circ} \mathrm{C}$ de precisión, fueron hechas en el momento de la toma de muestras.

Análisis de los sedimentos: En cada estación, con ayuda de un nucleador de $5 \mathrm{~cm}$ de diámetro, enterrado hasta una profundidad de $10 \mathrm{~cm}$ en el sedimento, se tomaron dos muestras de sedimento destinadas a los análisis granulométricos por tamizado a través de tamices de $2.00,1.00,0.50,0.25,0.125$ y $0.0625 \mathrm{~mm}$, según la metodología de Krumbein \& Pettijhon (1938). También se evaluó el contenido de material orgánica total por combustión a $550^{\circ} \mathrm{C}$ durante 24h (López-Jamar \& Cal 1990).

ANOVAS de dos vías fueron calculados para comparar la abundancia total, con datos transformados a $\log _{10}(n+1)$, entre estaciones y meses. La significancia estadística fue determinada por medio del Duncan LS mean test (Sokal \& Rohlf 1981). Se calculó el valor del índice de dominancia de la abundancia (McCloskey 1970), que toma en consideración para su cálculo el orden de abundancia de los grupos y su frecuencia de aparición en las muestras. Con el propósito de detectar posibles asociaciones entre los sitios de muestreo se realizó un análisis de conglomerados con la distancia euclidiana como medida de similitud y la unión completa como método de agrupamiento, utilizando el programa estadístico SYSTAT 12 (2007). Con el fin de observar posibles correlaciones entre los grupos de meiofauna y las características ambientales se realizó un análisis de componentes principales con la ayuda del programa STATGRAPHICS PLUS versión 5.1. Para ambos análisis se excluyeron los isópodos y nemertinos por presentar menos de $0.025 \%$ de la abundancia total.

\section{RESULTADOS}

Características ambientales: La distribución promedio mensual de la temperatura mostró el valor mínimo en febrero $\left(24.6^{\circ} \mathrm{C}\right)$ y el máximo en septiembre $\left(29.53^{\circ} \mathrm{C}\right)$, promedio $26.73^{\circ} \mathrm{C}$; desv. estándar 1.49 . Se observó un descenso progresivo de la temperatura desde octubre hasta enero-marzo y un incremento prácticamente constante desde este mes hasta septiembre (Fig. 1A). La salinidad presentó valores mínimos en septiembre (35.65) y máximos en febrero (36.73) y marzo (36.86), promedio=36.74; desv. estándar 0.08 . Se apreció un incremento paulatino de la salinidad desde octubre hasta febrero, y un descenso progresivo desde mayo hasta septiembre (Fig. 1A). Los sedimentos están constituidos casi exclusivamente por arena (96.62 a 97.68\%) (Fig. 1B). En la estación E1 los mayores porcentajes correspondieron a arena gruesa $(41.09 \%)$; en las estaciones E2 y E3 la arena gruesa y fina mostraron valores similares $(22.42 \%, 24.43 \%$ y $30.82 \%, 28.12 \%$, respectivamente) y, en la E4 predominaron las categorías de arena fina y media (34.81 y $28.76 \%$, respectivamente). El promedio de contenido de materia orgánica estuvo comprendido entre $0.61 \%$ (E2) y $0.85 \%$ (E3). Valores mínimos se presentaron en junio en la estación E1 (0.18\%) y máximos de $5.21 \%$ en noviembre (E1) y de $4.89 \%$ en septiembre (E3). 

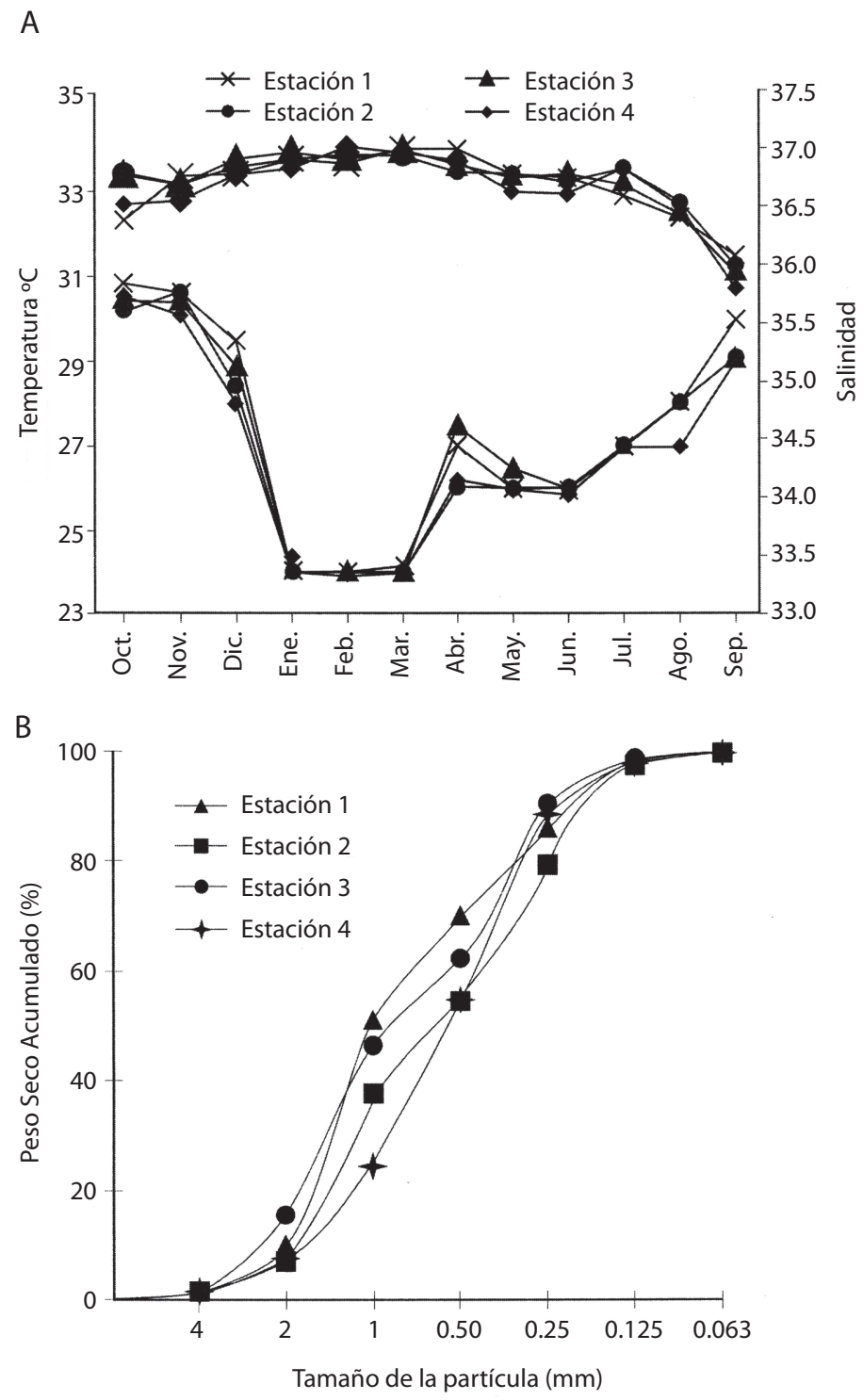

Fig. 1. (A) Valores mensuales de temperatura y salinidad en las estaciones. (B) Curvas acumulativas del porcentaje en peso de las categorías granulométricas de los sedimentos de las estaciones.

Fig. 1. (A) Monthly values of temperature and salinity at each station. (B) Cumulative weight percent curves of sediment granulometric categories of the stations.

Se recolectaron ejemplares pertenecientes a 14 táxones, los nemátodos fueron el grupo dominante $\left(58.36 \%\right.$; promedio $155.80 \mathrm{ind} . / 10 \mathrm{~cm}^{2}$; desv. estándar 29.07), seguidos por los ostrácodos $\left(17.10 \%\right.$; 45.60ind. $\left./ 10 \mathrm{~cm}^{2} ; 6.54\right)$ y copépodos harpacticoides $\left(15.72 \%\right.$; 41.96 ind. $/ 10 \mathrm{~cm}^{2}$; 17.39); estos tres grupos representaron $91.18 \%$ del total. Otros grupos representados fueron los tardígrados $(3.67 \%)$, poliquetos $(1.29 \%)$, gastrópodos $(1.06 \%)$, gastrotricos $(0.93 \%)$, oligoquetos $(0.75 \%)$, kinorrincos $(0.53 \%)$, halacáridos $(0.28 \%)$, pelecípodos $(0.25)$, turbeláridos (0.03), anfípodos $(0.02 \%)$, y nemertinos $(0.01)$. El análisis de varianza mostró diferencias de la 
abundancia de los táxones entre meses y estaciones $(p<0.001)$. Diferencias en la abundancia de individuos (test de intervalos múltiples) fueron observadas entre meses y estaciones. Las interacciones entre los dos factores fueron significativas ( $\mathrm{F}: 37.538, \mathrm{p}<0.001)$.

La densidad promedio mensual en las estaciones estuvo comprendida entre 173 (est. 4) y 449ind. $/ 10 \mathrm{~cm}^{2}$ en la est. 1 promedio 262.53 ; 134.18) (Fig. 2B). Las menores densidades se presentaron en las estaciones 1-3 en julio (70, 65 y 55 ind. $/ 10 \mathrm{~cm}^{2}$, respectivamente) y en la est. 4 en agosto $\left(45 \mathrm{ind} . / 10 \mathrm{~cm}^{2}\right)$. En la est. 1 se observaron los mayores valores de densidad en noviembre, diciembre, abril y septiembre $\left(855,991,703\right.$ y $716 \mathrm{ind} . / 10 \mathrm{~cm}^{2}$, respectivamente), los menores valores se presentaron en julio (69.73ind./10 $\mathrm{cm}^{2}$ ) y agosto $\left(158.54 \mathrm{ind} . / 10 \mathrm{~cm}^{2}\right)$, con un promedio mensual de 449ind./10 $\mathrm{cm}^{2}$; desv. estándar 300.16. En la estación 2 la densidad promedio fue 218ind. $/ 10 \mathrm{~cm}^{2}$; 161.81, con los mayores valores en octubre (559ind./10 $\mathrm{cm}^{2}$ ), enero (421ind. $/ 10 \mathrm{~cm}^{2}$ ) y junio (399ind. $/ 10 \mathrm{~cm}^{2}$ ), y los menores en febrero (55.26ind./10 $\mathrm{cm}^{2}$ ) y julio $\left(65.13\right.$ ind. $\left./ 10 \mathrm{~cm}^{2}\right)$. En la estación 3, el promedio mensual de la densidad fue de 211 ind. $/ 10 \mathrm{~cm}^{2}$; 110.93 ; las mayores densidades se presentaron en octubre, diciembre y junio $\left(355,344\right.$ y $317 \mathrm{ind} . / 10 \mathrm{~cm}^{2}$, respectivamente), y
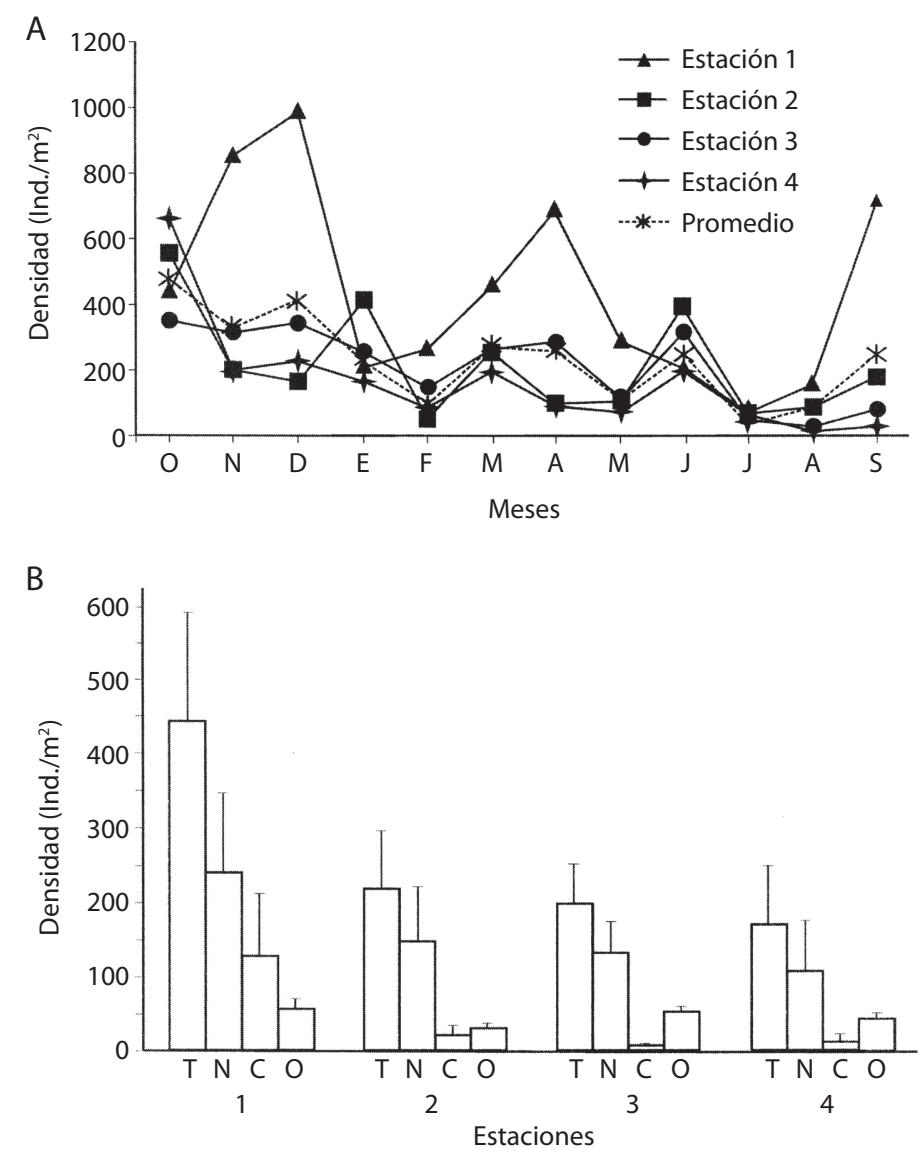

Fig. 2. (A) Promedios mensuales de las densidades de la meiofauna en las estaciones. (B) Densidad promedio total (T) y desviaciones estándar, de Nemátodos $(\mathbf{N})$, Copépodos $(\mathbf{C})$ y ostrácodos $(\mathbf{O})$ en cada estación.

Fig. 2. (A) Monthly average density of meiofauna on the stations. (B): Total average density (T) and standard deviation of meiofauna, Nematodes (N), Copepods (C) and Ostracods (O) at each station. 
las menores en julio (55ind. $/ 10 \mathrm{~cm}^{2}$ ) y en agosto (56 ind. $/ 10 \mathrm{~cm}^{2}$ ). La estación 4 fue la que mostró el menor promedio de densidad (173ind./10 $\mathrm{cm}^{2}$; 168.77), aunque en octubre presentó una densidad elevada (661ind. $\left./ 10 \mathrm{~cm}^{2}\right)$; excepto en este mes y en diciembre (234ind. $/ 10 \mathrm{~cm}^{2}$ ) en los demás meses la densidad fue inferior a 200ind. $/ 10 \mathrm{~cm}^{2}$ y de estos, seis meses mostraron densidades inferiores a $100 \mathrm{ind} . / 10 \mathrm{~cm}^{2}$ ).

Los promedios de las densidades mensuales (Fig. 2A) muestran valores altos de octubre (503ind./10 $\mathrm{cm}^{2}$ ) a diciembre, valores medios en marzo, abril, junio y septiembre, y bajos en los restantes meses, particularmente en febrero, julio y agosto (64 y 86 ind. $/ 10 \mathrm{~cm}^{2}$, respectivamente).

La distribución mensual de los grupos meiofaunísticos no mostró un patrón de comportamiento definido. En líneas generales, los picos de abundancia de los grupos más abundantes (nemátodos, copépodos harpacticoides y ostrácodos) se presentaron en meses y estaciones diferentes (Fig. 2B). Los nemátodos mostraron las mayores densidades en la estación 1 en noviembre (787ind. $/ 10 \mathrm{~cm}^{2}$ ) y en septiembre (512ind.$/ 10 \mathrm{~cm}^{2}$ ). En octubre fueron abundantes en las estaciones 2 y 4 (458 y $514 \mathrm{ind} . / 10 \mathrm{~cm}^{2}$, respectivamente) (Fig. 3A). Los copépodos
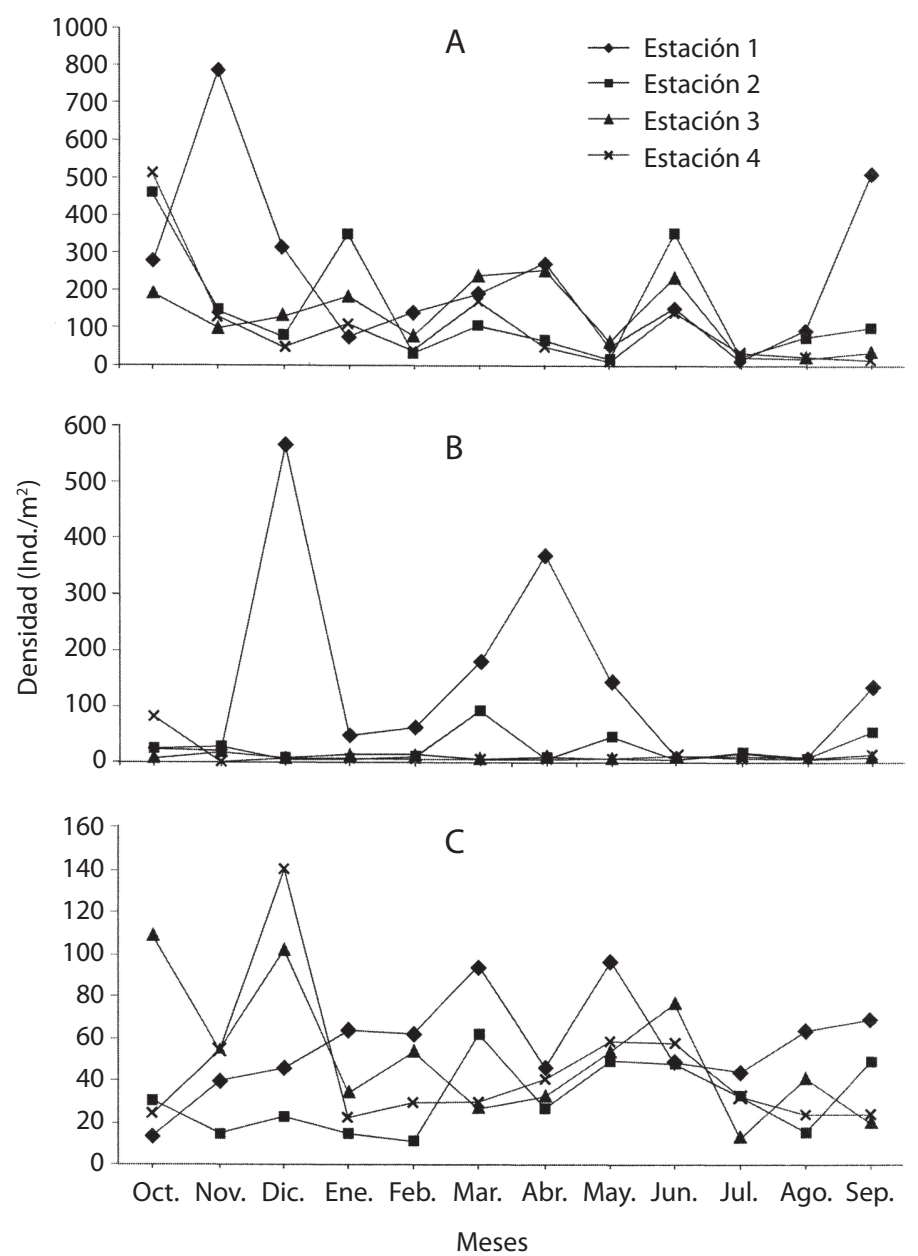

Fig. 3. Densidad promedio mensual en cada estación de nemátodos (A), copépodos (B) y ostrácodos (C). Fig. 3. Monthly average density at each station of nematodes (A), copepods (B) and ostracods (C). 
presentaron la máxima densidad en la estación 1 en diciembre $\left(565\right.$ ind. $\left./ 10 \mathrm{~cm}^{2}\right)$ y otro valor elevado (367ind. $/ 10 \mathrm{~cm}^{2}$ ) en la misma estación en abril; en la mayoría de las restantes estaciones $(64.58 \%)$ las densidades no alcanzaron 50ind./10 $\mathrm{cm}^{2}$ (Fig. 3B). La distribución mensual de las abundancias de ostrácodos es más compleja (Fig. 3C), con densidades comprendidas entre 13 (est. 1, oct.) y $140 \mathrm{ind} . / 10 \mathrm{~cm}^{2}$ (est. 4, dic.), y valores relativamente elevados en octubre en la est. 1 (109ind. $/ 10 \mathrm{~cm}^{2}$ ) y en diciembre en la estación 3 (102ind./10 $\mathrm{cm}^{2}$ ).

Las mayores densidades en la estación 1 durante noviembre y septiembre se debieron a la neta dominancia de nemátodos (591 y 384 ind.$/ 10 \mathrm{~cm}^{2}$, respectivamente), mientras que el pico observado en abril lo produjo la abundancia de copépodos $\left(275 \mathrm{ind} . / 10 \mathrm{~cm}^{2}\right)$ y el de diciembre fue ocasionado por los copépodos (424ind./10 $\mathrm{cm}^{2}$ ) y ostrácodos (105ind./10 $\mathrm{cm}^{2}$ ).

Los nemátodos fueron el grupo dominante seguidos por los ostrácodos y copépodos harpacticoides. Los nemátodos fueron el grupo más abundante en 35 de las 48 muestras, los ostrácodos en ocho muestras y los copépodos en dos; la segunda mayor abundancia fue compartida por los nemátodos (11) y copépodos (7) y la tercera mayor abundancia por nemátodos (2), ostrácodos (12) y copépodos (17). Nemátodos y ostrácodos estuvieron presentes en todas las muestras, mientras que los copépodos estuvieron ausentes en tres muestras.

En el dendrograma resultante del análisis de conglomerados (Fig. 4) se aprecia la formación de dos grupos principales de sitios de muestreo, A y B, constituido el primero por solo seis sitios y el grupo B por 42 que, a su vez, está conformado por dos subgrupos, B1 y B2, constituidos por 40 y 2 sitios de muestreo, respectivamente. A menor distancia, 11 sitios conforman el subgrupo B1a, y 29 el subgrupo B1b. A su vez, este subgrupo abarca dos conglomerados, uno $\left(\mathrm{B}_{1} \mathrm{~b}_{1}\right)$ con un número elevado de sitios de muestreo (27), y otro $\left(\mathrm{B} 1 \mathrm{~b}_{2}\right)$ constituido solo por dos sitios.

Los resultados del análisis de componentes principales (Fig. 5) muestran que las estaciones 3 y 4 aparecen correlacionadas negativamente con las estaciones 1 y 2 , y estas últimas muestran correlación entre sí. Los nemátodos y poliquetos mostraron estrecha correlación positiva con la arena fina, al igual, aunque en menor grado, que turbeláridos, halacáridos, kinorrincos, y gastrotricos, Los oligoquetos, gastrópodos y copépodos presentaron correlación positiva con la arena gruesa, mientras que los ostrácodos no mostraron correlación alguna.

En el cuadro 1 se presenta información sobre algunos aspectos metodológicos y resultados de densidades de varios estudios sobre meiofauna en zonas tropicales a templadas americanas.

\section{DISCUSIÓN}

Los valores de temperatura y salinidad se mantuvieron en intervalos relativamente estrechos. Las menores salinidades observadas en agosto y septiembre en la estación 4 se debieron al drenaje de agua dulce proveniente del río Tacal, de cuya desembocadura se encuentra próxima esta estación.

La comparación de los resultados entre estudios similares se dificulta debido a la ausencia de uniformidad en la metodología para la recolección y separación de la meiofauna, lo cual, en parte, ya fue señalado hace más de 40 años por McIntyre (1969) y Harris (1972). Son varios los factores que intervienen en el proceso de recolección y posterior análisis de las muestras de meiofauna, siendo los principales la técnica de separación de la meiofauna y el sedimento (flotación, decantación, centrifugado, tamizado, entre otros) el diámetro del nucleador, la profundidad de penetración del mismo y los tamices para separar la meiofauna de la macrofauna y de la microfauna. En estudios similares, realizados en áreas tropicales a templadas de la costa americana, se advierten diferencias en algunos aspectos metodológicos. Así, con relación al diámetro interno de los nucleadores, se aprecia un intervalo desde 2 hasta $4.4 \mathrm{~cm}$. La profundidad de penetración del nucleador varía de 4 a $30 \mathrm{~cm}$. Para la separación de la meiofauna y macrofauna se utilizan tamices (superiores) 


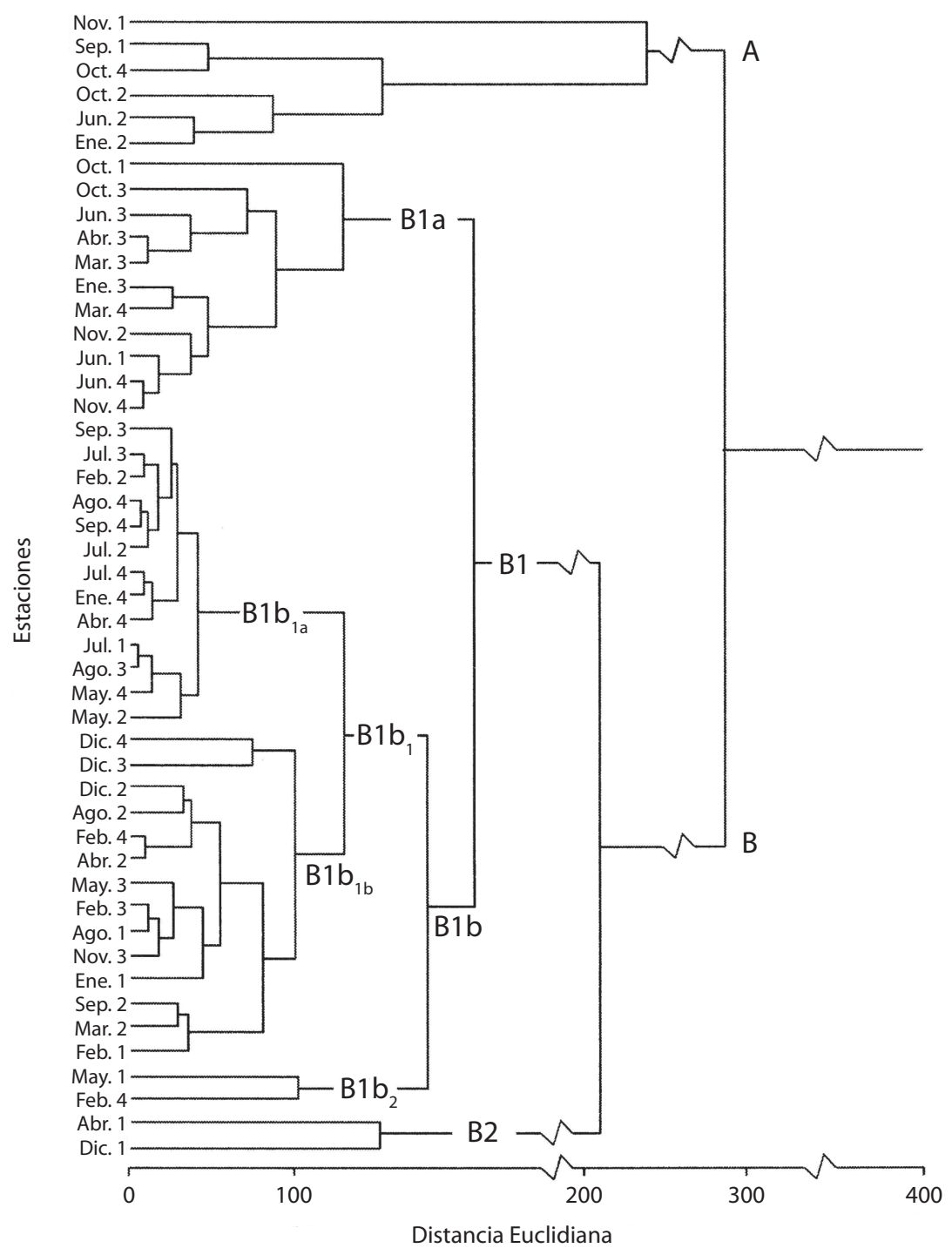

Fig. 4. Dendrograma obtenido por el método de uniones completas basado en la distancia Euclidiana. Meses en abreviaturas y estaciones en números.

Fig. 4. Dendrogram obtained by the complete linkage method bassed on the Euclidean distance. Months in abbreviatures and stations in numbers.

desde 175 hasta $1000 \mu \mathrm{m}$ y para la separación de la meiofauna y la microfauna tamices (inferiores) desde 32 hasta $100 \mu \mathrm{m}$. La variedad de tamices utilizados muestra que no existe uniformidad ni siquiera en la definición del término meiofauna. Con relación a esto Soltwedel (1997) señaló que es urgente la estandarización de los tamaños de los tamices. La importancia de la selección del tamiz para la extracción de la meiofauna fue analizada por de Bovée et al. (1974), quienes mostraron que el porcentaje de retención de organismos se incrementa con la reducción del tamaño de la malla.

Los resultados de las densidades de la meiofauna se expresan en abundancia por unidad de superficie, aun cuando las muestras 


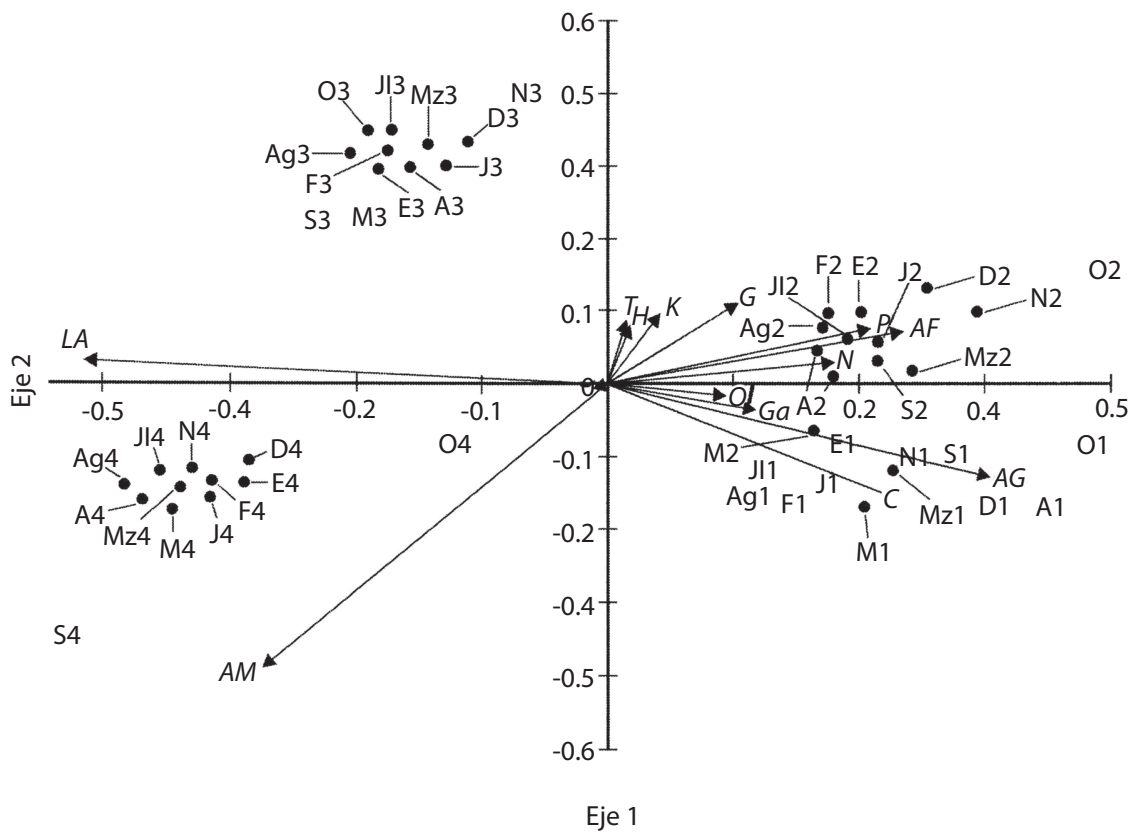

Fig. 5. Proyección de localidades de muestreo, categorías granulométricas de los sedimentos y abundancia de táxones en los dos primeros ejes del análisis de componentes principales. Los meses y estaciones de muestreo están representados por la inicial del mes seguida del número de la estación, ej.: O3, significa Octubre en la estación 3. Granulometría: (AG) Arena Gruesa (AM) Arena Media (AF) Arena Fina (LA) Limo-Arcilla. Táxones: (C) Copépodos (G) Gastrotricos, (Ga) Gastrópodos (H) Halacáridos (K) Kinorrincos (N) Nemátodos (O) Ostrácodos (Ol) Oligoquetos (P) Poliquetos (T) Turbelarios.

Fig. 5. Projection of sampling sites, granulometric categories of the sediments and taxa abundance on the two first axes determined by the principal components analysis. Sampling months and stations are represented by the initial of the month and the station number, example: O3, means October at station 3. Granulometry: (AG) Coarse sand (AM) Median sand (AF) Fine sand (LA) Silt and clay. Taxa: Copepods (G) Gastrotrichs (Ga) Gastropods (H) Halacarids (K) Kinorhynchs (N) Nematodes (O) Ostracods (OI) Oligochaetes (P) Polychaetes (T) Turbellarians.

corresponden a volúmenes. Como es razonable suponer, a mayor profundidad de penetración del nucleador en el sedimento la posibilidad de recolectar un mayor número de organismos se incrementa; por lo cual, expresar densidades por unidad de superficie, cuando se comparan muestras de diferentes volúmenes, puede conducir a interpretaciones equívocas.

Teniendo presente estas consideraciones, se hacen algunas comparaciones entre los valores obtenidos en este trabajo y los resultados de otros estudios. La densidad promedio de la meiofauna en la playa San Luis (263ind./10 $\mathrm{cm}^{2}$ ) es baja si se compara con otros estudios realizados en zonas tropicales americanas (Cuadro 1); densidades de meiofauna en otras áreas del planeta son ofrecidas por McIntyre (1969) y Alongi (1989). Valores similares a los obtenidos en este estudio fueron registrados por Decho et al. (1985) en Florida (309ind./10 $\mathrm{cm}^{2}$ ). En una laguna costera del Golfo de California, Gómez-Noguera \& Hendrickx (1997) obtuvieron densidades de 50 a 2676.83 ind. $/ 10 \mathrm{~cm}^{2}$ (promedio $425.53 \mathrm{ind} . / 10 \mathrm{~cm}^{2}$ ), presentándose las mayores densidades en playas fangosas afectadas por drenajes agro-industriales con elevados contenidos de carbono y nitrógeno y las menores en estaciones arenosas con bajos contenidos de esos elementos. Al calcular el promedio sin tomar en consideración el extremado valor obtenido en una de esas estaciones, la densidad fue de $264.72 \mathrm{ind} . / 10 \mathrm{~cm}^{2}$, muy 
CUADRO 1

Datos acerca de la toma de muestras, separación de la meiofauna, número de grupos $(\mathrm{N})$ y densidad (ind. $/ \mathrm{m}^{2}$ ) de la meiofauna en algunas localidades tropicales americanas*

TABLE 1

Data about sampling, processing, number of taxa and of organisms and density (ind. $/ \mathrm{m}^{2}$ ) of meiofauna from some tropical American sites*

\begin{tabular}{|c|c|c|c|c|c|c|c|}
\hline $\mathrm{D}$ & Pr. & T. S. & T. I. & $\mathrm{N}$ & Densidad & Referencia & Localidad \\
\hline 3.5 & 10 & 500 & 62 & & $123-1334(570)$ & Coull, 1970 & Bermuda \\
\hline 2.6 & 6 & & & 18 & 634 & Decho et al. 1985 & Florida \\
\hline 1.3 & 6 & 500 & 62 & 16 & $117-425(217)$ & De la Cruz \& Vargas 1987 & Costa Rica \\
\hline 1.3 & 6 & 500 & 63 & 13 & 2400 & Vargas 1988 & Costa Rica \\
\hline 2.69 & 12 & 250 & 40 & $>11$ & $678-6989(2260)$ & Boucher \& Gourbault 1990 & Guadalupe \\
\hline 3 & 2 & 500 & 63 & 18 & $50-2676.83(426)$ & Gómez-Noguera \& Hendrickx 1997 & México \\
\hline 3.57 & 20 & 1000 & 100 & & $110-4191$ & López-Cánovas \& Lalana 2001 & Cuba \\
\hline 2.7 & 5 & & 63 & 11 & 2688 y 10250 & Gomes et al. 2002 & Brasil \\
\hline 4.4 & 10 & & 45 & 9 & $27-398$ & Sandulli \& Grimaldi 2002 & Albania \\
\hline 2.8 & 10 & 500 & 100 & 15 & $98.3-748.5$ & Armenteros et al. 2003 & Cuba \\
\hline 2.5 & 10 & & 63 & 9 & $77-1589$ & Netto \& Gallucci 2003 & Brasil \\
\hline 2.5 & & 500 & 37 & 13 & 892.12-7 133.5 (3 933) & Pérez \& López-Cánovas 2006 & Cuba \\
\hline 3.5 & 30 & & 45 & & $1556-13125$ & Albuquerque et al. 2007 & Brasil \\
\hline 2.6 & 10 & 500 & 45 & 4 y 5 & & Armenteros et al. 2008 & Cuba \\
\hline 2.9 & 6 & 500 & 50 & 12 & $415.2-1212.5$ & Díaz-Asencio et al. 2009 & Cuba \\
\hline 2.6 & 5 & 500 & 63 & 22 & $1056-2333$ & Edwards 2009 & Jamaica \\
\hline 2.5 & 4 & 500 & 64 & 17 & $518-1209$ & Vásquez-Suárez et al. 2010 & Venezuela \\
\hline 2.5 & 10 & 500 & 63 & 14 & $45-991(263)$ & Este estudio & Venezuela \\
\hline
\end{tabular}

*(D: Diámetro del nucleador, Pr.: Profundidad de penetración del nucleador en el sedimento, T.S.: Tamiz superior, T.I.: Tamiz inferior, N: número de grupos)/ (D: Diameter of corer, Pr.: Penetration depth of corer into sediment, T.S.: Superior sieve, T.I.: Inferior sieve, N: number of taxa).

similar a la obtenida en la Playa de San Luis, con sedimentos arenosos y alejada de zonas agrícolas. Las bajas densidades relativas obtenidas en este estudio estarían relacionadas con la granulometría de los sedimentos, constituidos principalmente por arena media a gruesa. Valores de densidad bajos, similares a los obtenidos en este estudio, logrados por Decho et al. (1985), Armenteros et al. (2003) y VásquezSuárez et al. 2010) fueron asimismo obtenidos en playas arenosas. Wieser (1959) manifiesta que el tamaño de las partículas puede afectar la distribución de los organismos, y que sedimentos con un diámetro promedio de $200 \mu \mathrm{m}$ constituyen una barrera para ciertos grupos.

El número de grupos meiofaunísticos recolectados en este estudio (14) está entre los más elevados señalados en otras investigaciones realizadas en zonas tropicales y subtropicales (Cuadro 1). Generalmente, como en este estudio, el grupo dominante corresponde a nemátodos seguido por copépodos u ostrácodos. Sin embargo, en algunos casos, la mayor abundancia la presentan otros grupos. Así, por ejemplo, en fondos de arrecifes coralinos de Isla del Caño, Costa Rica, el grupo dominante fue el de los foraminíferos, seguidos muy de cerca por copépodos y nemátodos (Guzmán-Espinal et al. 1987). Los tardígrados fueron el grupo netamente dominante seguidos por nemátodos y copépodos en una playa arenosa de Brasil, atribuyéndose la elevada densidad de tardígrados a la buena oxigenación de los sedimentos (Albuquerque et al. 2007).

El comportamiento de las densidades mensuales de nemátodos en la playa San Luis 
concuerda con los resultados obtenidos por Albuquerque et al. (2007) y por Chindah \& Braide (2001), mientras que el de los copépodos contrasta con lo manifestado por estos autores, al señalar que nemátodos y copépodos son más abundantes en los meses lluviosos y contradice lo observado por Guzmán-Espinal et al. (1987), quienes observaron que estos grupos son más numerosos en la época de sequía.

La evolución del promedio de la densidad mensual no permite apreciar una clara relación con las condiciones climáticas, aunque se observó una tendencia a la reducción de la densidad desde octubre hasta febrero, esto es, desde el final de la época de lluvias hasta la parte media de la época de sequía, y los valores más bajos en julio y agosto, en pleno periodo lluvioso, lo cual se asemeja parcialmente a los resultados obtenidos por Chindah \& Braide (2001), quienes manifiestan que las mayores abundancias de la meiofauna se presentaron en el periodo seco; aunque señalan que algunos táxones fueron abundantes en un periodo mientras otros lo fueron en otro, relacionando esas diferencias con el incremento de nutrientes durante la estación sea y con interacciones inter e intraespecíficas. Por su parte, Coull (1970) manifestó que en regiones tropicales se puede apreciar cierta estacionalidad, con las mayores abundancias durante el periodo más cálido. En la playa de San Luis, la mayor abundancia de nemátodos se presentó en la estación 4 en noviembre, final del periodo cálido y lluvioso, mientras que los copépodos mostraron las mayores abundancias en diciembre y abril, meses secos y de temperaturas más bajas.

La estrecha amplitud de los valores de temperatura y salinidad en la playa San Luis a lo largo del año, el intervalo relativamente angosto del tamaño de las partículas de los sedimentos y del contenido de materia orgánica en las diferentes estaciones y meses, no parece provocar un efecto notable sobre las poblaciones de la meiofauna; aunque el dendrograma del análisis de conglomerados (Fig. 4) muestra que en el conglomerado $\mathrm{B} 1 \mathrm{~b}_{1 \mathrm{a}}$ existe un predominio de muestras tomadas durante los meses de lluvia; mientras que en el conglomerado
$\mathrm{B} 1 \mathrm{~b}_{1 \mathrm{~b}}$ el predominio corresponde a muestreos realizados mayormente en meses de sequía. Las características granulométricas de los sedimentos representan un factor de gran importancia en la abundancia y distribución de la meiofauna (Giere 1993, Rodríguez et al. 2001). En este estudio la preferencia de los nemátodos por arenas finas y la de los copépodos por arena gruesa, evidenciadas por el análisis de componentes principales, ratifica lo señalado, entre otros, por Amjad \& Gray (1983) y Raffaelli (1987). Por consiguiente, en la playa de San Luis el control de las poblaciones meiofaunísticas estaría compartido por las condiciones climáticas y por los aspectos de la biología de las especies, puesto que la interfase agua-sedimento es un ambiente dinámico donde se producen cambios en los procesos físicos, químicos y biológicos, que afectan a las comunidades de la meiofauna, por lo que el estudio de un factor no puede explicar la distribución de los grupos meiofaunísticos (Albuquerque et al. 2007).

A diferencia de la mayoría de los estudios, en los que la meiofauna está netamente dominada por los nemátodos (Escaravage et al. 1989, Kennedy \& Jacoby 1997, Gomes et al. 2002, Gheskiere et al. 2005, Díaz-Asencio et al. 2009, Vásquez-Suárez et al. 2010), en la playa San Luis la dominancia de ese grupo es compartida, en algunos meses y estaciones, con los ostrácodos y, en menor grado, con los copépodos. En sedimentos finos, la abundancia de nemátodos se incrementa y la de los copépodos disminuye; mientras que en sedimentos arenosos ocurre lo contrario (Amjad \& Gray 1983). En este estudio, realizado en una playa arenosa, era de esperarse que los copépodos fueran abundantes; sin embargo, ocuparon el tercer lugar en orden de densidad, precedidos por los ostrácodos, similar a los resultados obtenidos en sedimentos finos por De La Cruz \& Vargas (1987) y Vargas (1988) en el Golfo de Nicoya, Costa Rica. De acuerdo a Hulings (1971), la abundancia y distribución de los ostrácodos están controladas principalmente por la disponibilidad de alimento, tipo de substrato y época del año. Por su parte, Wieser (1959) manifiesta que estos organismos son 
dominantes en sedimentos con un diámetro promedio menor de $150 \mu \mathrm{m}$ y no se presentan en sedimentos con partículas de más de $260 \mu \mathrm{m}$; sin embargo, McIntyre (1969) sostiene que en sedimentos arenosos submareales los ostrácodos son más numerosos que en sedimentos fangosos. En la playa San Luis, las estaciones que mostraron mayor abundancia de ostrácodos fueron la 1 y la 3 , la primera caracterizada por un mayor porcentaje de arena gruesa, y la segunda por porcentajes similares de arena gruesa y fina, lo cual contradice lo señalado por Wieser (1959) y apoya lo manifestado por McIntyre (1969). En la playa estudiada son frecuentes las acumulaciones de macroalgas, principalmente Ulva spp. y Enteromorpha spp., lo que podría incrementar la abundancia de ostrácodos (Tietjen 1969, Hull 1997).

Las variaciones de la abundancia de los grupos meiofaunales en los diferentes meses y estaciones podrían deberse, por un lado, a la distinta duración y aparición de los periodos reproductivos y de reclutamiento de las especies y, por otro, a la disponibilidad y calidad del alimento (Gomes et al. 2002), a la depredación inter e intraespecífica (Watzin 1985), a la perturbación (Gheskiere et al. 2005), entre otros. En este estudio la identificación se hizo a nivel de grandes grupos, por lo que se desconoce la riqueza específica de cada grupo en las estaciones y meses. Es muy probable que las especies de cada grupo muestren diferencias en la época de reproducción, en el número de periodos reproductivos y en la duración de los ciclos de vida, lo cual explicaría la ausencia de un patrón más definido de la distribución mensual de los grupos meiofaunales. A esta situación contribuye el angosto intervalo de los valores de los parámetros ambientales tomados en consideración (temperatura, salinidad, granulometría y contenido de materia orgánica).

Las actividades relacionadas con el turismo afectan la diversidad de la nematofauna en playas arenosas y su efecto perturbador es parecido al producido por las corrientes y el oleaje, factores que afectan negativamente la abundancia de la meiofauna (Schratzberger \& Warwick 1998, Dernie et al. 2003, Gheskiere et al. 2005, Díaz-Asencio et al. 2009). En la playa San Luis la perturbación antrópica originada por los usuarios de este balneario, que alteran profundamente los sedimentos superficiales y modifican la zonación vertical de la meiofauna, contribuye a la ausencia de un patrón en la distribución de la meiofauna. Este balneario es frecuentado los días no laborables, y durante las vacaciones escolares (julio a septiembre) $\mathrm{y}$, precisamente, es en julio y agosto cuando se presentaron los valores más bajos de abundancia.

\section{AGRADECIMIENTOS}

Se agradece a los revisores anónimos los valiosos consejos que permitieron mejorar el manuscrito.

\section{RESUMEN}

La meiofauna incluye metazoos y foraminíferos bénticos que pasan a través de un tamiz de $500 \mathrm{~mm}$ y son retenidos en malla de $40-63 \mu \mathrm{m}$. Se estudió la variación espacial y temporal de la meiofauna en cuatro estaciones submareales a $1 \mathrm{~m}$ de profundidad, de una playa arenosa situada en la costa nororiental de Venezuela. Mensualmente, durante un año, en cada estación, con un nucleador de $2.5 \mathrm{~cm}$ de diámetro interno, se tomaron tres réplicas de sedimento hasta $10 \mathrm{~cm}$ de profundidad y se pasaron a través de un tamiz de $0.063 \mathrm{~mm}$ de abertura de malla. Se identificaron 14 grupos meiofaunales, excluidos los foraminíferos, siendo los nemátodos, ostrácodos y copépodos harpacticoides los más abundantes. La densidad mensual estuvo comprendida entre 64 y 503 ind. $/ 10 \mathrm{~cm}^{2}$, y la densidad media en las estaciones entre 173 y 449 ind.$/ 10 \mathrm{~cm}^{2}$. La densidad de la meiofauna en la playa San Luis es baja en comparación con otros estudios realizados en zonas tropicales. En esta playa el control de las comunidades meiofaunísticas es compartido por las condiciones climáticas y la biología de las especies.

Palabras clave: meiofauna, nemátodos, copépodos, ostrácodos, Caribe, Venezuela.

\section{REFERENCIAS}

Alongi, D.M. 1989. Ecology of soft-bottom benthos: a review with emphasis on emerging concepts. Rev. Biol. Trop. 37: 85-100.

Albuquerque, E.F., A.P. Brandão Pinto, A. d'Alcântara De Queiroz \& V. Gomes. 2007. Spatial and temporal 
changes in interstitial meiofauna on a sandy ocean beach of South America. Braz. J. Oceanogr. 55: 121-131.

Amjad, S. \& J. Gray. 1983. Use of the nematode-copepod ratio as an index of organic pollution. Mar. Pollut. Bull. 14: 178-181.

Andersen, J.H., L. Schlüter \& G. Aertebjerg. 2006. Coastal eutrophication: recent developments in definitions and implications for monitoring strategies. J. Plankton Res. 28: 117-143.

Armenteros, M., G. González-Sansón \& R. Lalana. 2003. Composición y abundancia del meiobentos en un sector sublitoral de ciudad de La Habana, Cuba. Rev. Inv. Mar. 24:3-10

Boucher, G. \& N. Gourbault. 1990. Sublitoral meiofauna and diversity of nematode assemblages off Guadeloupe Islands (French West Indies). Bull. Mar. Sci. 47: 448-463.

Brazeiro, A. 2001. Relationship between species richness and morphodynamics in sandy beaches: what are the underlying factors? Mar. Ecol. Prog. Ser. 224: 35-44.

Buffan-Dubau, E., R. Wit \& J. Castel. 1996. Feeding selectivity of the harpacticoid copepod Canuella perplexa in benthic muddy environments demonstrated by HPLC analyses of chlorin and carotenoid pigments. Mar. Ecol. Prog. Ser. 137: 71-82.

Chindah, A.C. \& S.A. Braide. 2001. Meiofauna occurrence and distribution in different substrate types of bonny brackish wetland of the Niger Delta. J. Appl. Sci. Environ. Manag. 5: 33-41.

Coull, B.C. 1970. Shallow water meiobenthos off the Bermuda platform. Oecologia, Berlin 4: 325-357.

Covazzi, A., A. Pusceddu, N. Della Croce \& R. Danovaro. 2001. Spatial and temporal changes in beach meiofaunal communities of the Ligurian Sea (NW Mediterranean). Rev. Biol. Mar. Oceanol. 35: 57-64.

De Bovée, F., J. Soyer \& P.H. Albert. 1974. The importance ogf the mesh size for the extraction of the muddy bottom meiofauna. Limnol. Oceanogr. 19: 350-254.

De La Cruz, E. \& J.A. Vargas. 1986. Estudio preliminar de la meiofauna de la playa fangosa de Punta Morales, Golfo de Nicoya, Costa Rica. Brenesia 25/26: 89-97.

De La Cruz, E. \& J.A. Vargas. 1987. Abundancia y distribución vertical de la meiofauna en la playa fangosa de Punta Morales, Golfo de Nicoya, Costa Rica. Rev. Biol. Trop. 35: 363-367.

Decho, A.W., W.D. Hummon \& J.W. Fleeger. 1985. Meiofauna-sediment interactions around subtropical seagrass sediments using factor analysis. J. Mar. Res. 43: 237255 .

Dernie, K.M., M.J. Kaiser, E.A. Richardson \& R.M. Warwick. 2003. Recovery of soft sediment communities and habitats following physical disturbance. J. Exp. Mar. Biol. Ecol. 285/286: 415-434.

Díaz-Asencio, L., M. Armenteros, M. Díaz-Asencio, R. Fernández-Garcés, M. Gómez-Batista \& C. AlonsoHernández. 2009. Variaciones espaciales y temporales de las comunidades de la meiofauna en la bahía de Cienfuegos, Cuba. Rev. Biol. Mar. Oceanogr. 44: 1322.

Edwards, C. 2009. Meiobenthos of The Discovery Bay Lagoon, Jamaica, with an emphasis on nematodes. $\mathrm{Ph} . \mathrm{D}$. Thesis, University of London, England (Consultado: 8 diciembre 2011, https://qmro.qmul.ac.uk/ xmlui/bitstream/handle/123456789/522/EDWARDSMeiobenthos2009.pdf? sequence $=1$.

Escaravage, V., M.E. García \& J. Castel. 1989. The distribution of meiofauna and its contribution to detritic pathways in tidal flats (Arcachon Bay, France). Sci. Mar. 53: 551-559.

Essink, K. \& H. Keidel. 1998. Changes in estuarine nematode communities following a decrease of organic pollution. Aquat. Ecol. 32: 195-202.

Fisher, R. \& M.J. Sheaves. 2003. Community structure and spatial variability of marine nematodes in tropical Australian pioneer seagrass meadows. Hidrobiologia 495: 143-158.

Fraschetti, S., C. Gambi, A. Giangrande, L. Musco, A. Terlizzi \& R. Danovaro. 2006. Structural and functional response of meiofauna rocky assemblages to sewage pollution. Mar. Poll. Bull. 52: 540-548.

Gheskiere, T., M. Vincx, J.M. Weslawski, F. Scapini \& S. Degraer. 2005. Meiofauna as descriptor of tourism induced changes at sandy beaches. Mar. Environ. Res. 60: 245265.

Giere, O. 1993. Meiobenthology: The Microscopic Fauna in Aquatic Sediments. Springer Verlag, Berlin, Alemania.

Gomes, C.C.A., P.J. Parreira, T.N. Cavalcanti, J. Souto \& L. Pereira. 2002. Variação temporal da meiofauna em área de manguezal em Itamaracá-Pernambuco. Atlântica 24: 89-96.

Gómez-Noguera, S.E. \& M.E. Hendricks. 1997. Distribution and Abundance of Meiofauna in a Subtropical Coastal Lagoon in the South-eastern Gulf of California. Mar. Poll. Bull. 34: 582-587.

Gray, J.S., R.S.S. Wu \& Y.Y. Or. 2002. Effects of hypoxia and organic enrichment on the coastal marine environment. Mar. Ecol. Progr. Ser. 238: 249-279.

Guiral, D., N. Gourbault \& M.N. Helleouet. 1995. Étude sédimentologique et méiobenthos d'un écosystème lagunaire modifié par un récif artificial à vocation aquacole: 1'acadja. Oceanol. Acta 18: 543-555.

Guzmán-Espinal, H.M., V. Ocando-Acuña \& J. CortésNúñez. 1987. Meiofauna associated with a Pacific coral reef in Costa Rica. Coral Reefs 6: 107-112. 
Harris, R.P. 1972. The distribution and ecology of the interstitial meiofauna of a sandy beach al Whitsand Bay, East Cornwall. J. Mar. Biol. Ass. U.K. 52: 1-18.

Herrera, A. \& J. Sánchez. 1982. Características de la meiofauna bentónica en la Bahía de Cienfuegos y algunos aspectos de su ecología. Cienc. Biol. 7: 13-32.

Hulings, N.C. 1971. Summary and current status of the taxonomy and ecology of benthic Ostracoda including interstitial forms. Smithson. Contr. Zool. 76:91-96.

Hull, S.L. 1997. Seasonal changes in diversity and abundances of ostracods on four species of intertidal algae with differing structural complexity. Mar. Ecol. Prog. Ser. 161: 71-82.

Iabichella, A.M. 1993. Evaluación bacteriológica del sector marino-costero San Luis-Guapo, Cumaná, Venezuela, según los criterios para aguas de contacto humano total y parcial. Tesis de Maestría, Universidad de Oriente, Cumaná, Venezuela.

Kennedy, A.D. \& C.A. Jacoby. 1999. Biological indicators of marine environmental health: Meiofauna-A neglected benthic component? Envir. Monit Assess. 54: 47-68.

Krumbein, W.C. \& F.J. Pettijhon. 1938. Manual of sedimentary petrology. Appleton-Century Co., Nueva York, Nueva York, EEUU.

La Rosa, T., S. Mirto, A. Mazzola \& R. Danovaro. 2001. Differential responses of benthic microbes and meiofauna to fish-farm disturbance in coastal sediments. Envir. Pollut. 112: 427-432.

López-Cánovas, C.I. \& R. Lalana. 2001. Benthic meiofauna distribution at three coral reefs from SW of Cuba. Rev. Invest. Mar. 22: 199-204.

López-Jamar, E. \& R.M. Cal. 1990. El sistema bentónico de la zona submareal de la ría de Vigo. Macroinfauna y microbiología del sedimento. Bol. Inst. Esp. Oceanogr. 6: 49-60

Mahmoudi, E., N. Essid, H. Beyrem, A. Hedfi, F. Boufahja, P. Vitiello \& P. Aissa. 2005. Effects of hydrocarbon contamination on a free living marine nematode community: Results from microcosm experiments. Mar. Pollut. Bull. 50: 1197-1204.

McCloskey, L. 1970. The dynamics of community associated with a marine scleractinian coral. Int. Rev. Hidobiol. 55: 13-81.

Mcintyre, A.D. 1969. Ecology of Marine Meiobenthos. Biol. Rev. 44: 245-290.

McLachlan, A. 1983. Sandy beach ecology-a review, p. 321-380. In A. McLachlan \& T. Erasmus (eds.) Sandy beach as ecosystems. La Haya, Holanda.

Netto, S. \& F. Gallucci. 2003 Meiofauna and macrofauna communities in a mangrove from the Island of Santa Catarina, South Brazil. Hydrobiologia 505: 159-170.
Ólafsson, E. \& R. Elmgren. 1991. Effects of biological disturbance by benthic amphipods Monoporeia affinis on meiobenthic community structure: a laboratory approach. Mar. Ecol. Progr. Ser. 74: 99-107.

Pérez, H.A. \& C. López-Cánovas. 2006. Distribución espacio-temporal de la meiofauna béntica en cuatro playas del Litoral Norte de La Habana. Rev. Biol. Trop. 54: 985-995.

Pfannkuche, O. \& H. Thiel. 1988. Sample processing, p. 134-145. In R.P. Higgins \& H. Thiel (eds.). Introduction to the study of meiofauna. Smithsonian Institution, Londres, Inglaterra.

Quintero, A., J. Bonilla, L. Serrano, M. Amaro, B. Rodríguez, G. Terejova \& Y. Figueroa. 2004. Características ambientales de la Bahía de Mochima y adyacencias de la Cuenca de Cariaco, Venezuela. Bol. Inst. Oceanogr. Venezuela, Univ. Oriente 43: 49-64.

Raffaelli, D. 1987. The behaviour of the Nematode/ Copepod ratio in organic pollution studies. Marine Environ. Res. 23: 135-152.

Rafaelli, D. \& C.F. Mason. 1981. Pollution monitoring with meiofauna, using the ratio of nematodes to copepods. Mar. Pollut. Bull. 12: 158-163.

Renaud-Mornant, J. \& N. Gourbault. 1984. Premières prospections faunistiques en Guadeloupe. II. Communautés des sables littoraux. Hydrobiology 118: 113-118.

Rodil, L.F. \& M. Lastra. 2004. Environmental factors affecting benthic macrofauna along a gradient of intermediate sandy beaches in northern Spain. Estuar. Coast. Shelf Sci. 61: 37-44.

Rodríguez, J.G., J. López \& E. Jaramillo. 2001. Community structure of the intertidal meiofauna along a gradient of morphodynamic sandy beach types in southern Chile. Rev. Chil. Hist. Nat. 74: 885-897.

Rosa, L.C. \& C.E. Bemvenuti. 2005. Effects of the burrowing crab Chasmagnathus granulate (Dana) on meiofauna of estuarine intertidal habitats of Patos Lagoon, Southern Brazil. Braz. Arch. Biol. Technol. 48: 267274 .

Sandulli, R. \& S. De Zio Grimaldi. 2000. Status of intertidal meiofauna along an Albanian coastline exposed to the possible influence of Durres sewer. Biol. Mar. Medit. 7: 731-733.

Schratzberger, M. \& R.M. Warwick. 1998. Effects of physical disturbance on nematode communities in sand and mud: a microcosm experiment. Mar. Biol. 130: 643-650.

Smol, N., R. Huys \& M. Vincx. 1991. A 4-years' analysis of the meiofauna community of a dumping site for $\mathrm{TiO}^{2-}$ waste off the Dutch coast. Chem. Ecol. 5: 197-215.

Sokal, R.R. \& F.J. Rohlf. 1981. Biometry, the principles and practice of statistics in biological research. W.H. Freeman, San Francisco, EEUU. 
Soltwedel, T. 1997. Meiobenthos distribution pattern in the tropical East Atlantic: indication for fractioned sedimentation of organic matter to the sea floor? Mar. Biol. 129: 747-756.

Sutherland, T.F., C.D. Levings, P. Petersen \& Y.B. Piercey. 2007. The use of meiofauna as indicators of benthic organic enrichment associated with salmonid aquaculture. Mar. Pollut. Bull. 54: 1249-1261.

Terejova, G., A. Quintero \& A. Piñero. 1998. Descripción de las características climáticas de Carúpano, Estado Sucre (Venezuela). Bol. Inst. Oceanogr. Venezuela, Univ. Oriente 37: 43-52.

Tietjen, J.H. 1969. The ecology of shallow water meiofauna in two New England estuaries. Oecologia 2: 251-291.

Tietjen, J.H. 1984. Distribution and species diversity of deep-sea nematodes in the Venezuela Basin. Deepsea Res. 31: 119-132.
Vargas, J.A. 1988. A survey of the meiofauna of a eastern tropical Pacific intertidal mud flat. Rev. Biol. Trop. 36: $541-544$

Vásquez-Suárez, A., M. González, O. Díaz \& I. LiñeroArana. 2010. Variación temporal de la meiofauna en sedimentos del sistema lagunar "Laguna de Raya", Estado Nueva Esparta, Venezuela. Interciencia 35: 1-7.

Warwick, R.M., K.R. Clarke \& J.M. Gee. 1990. The effects of disturbance by soldier crabs Mictyris platycheles H. Milne Edwards on meiobenthic community structure. J. Exp. Mar. Biol. Ecol. 135: 19-33.

Watzin, M.C. 1985. Interactions among temporary and permanent meiofauna: observations on the feeding and behavior of selected taxa. Biol. Bull. 169: 397-416.

Wieser, W. 1959. The effect of grain size on the distribution of small invertebrates inhabiting the beaches of Puget Sound. Limnol. Oceanogr. 4: 181-194. 
